\title{
Label-retention expansion microscopy
}

Xiaoyu Shi1,8, Qi Li ${ }^{1,2,8}$, Zhipeng Dai³, Arthur A. Tran4, Siyu Feng ${ }^{5}$, Alejandro D.

Ramirez ${ }^{1}$, Zixi Lin ${ }^{1}$, Xiaomeng Wang ${ }^{1}$, Tracy T. Chow ${ }^{6}$, Ian B. Seiple ${ }^{1,2,{ }^{*}}$, Bo Huang ${ }^{1,6,7,{ }^{*}}$

1. Department of Pharmaceutical Chemistry, University of California, San Francisco, San

Francisco, CA 94143 USA

2. Cardiovascular Research Institute, University of California, San Francisco, San

Francisco, CA 94143 USA

3. Department of Bioengineering and Therapeutic Sciences, University of California, San

Francisco, San Francisco, CA 94143 USA

4. Graduate Program in Chemistry and Chemical Biology, University of California, San

Francisco, San Francisco, CA 94143 USA

5. UC Berkeley - UCSF Joint Graduate Program in Bioengineering, University of

California, San Francisco, San Francisco, CA 94143 USA

6. Department of Biochemistry and Biophysics, University of California, San Francisco, San Francisco, CA 94143 USA

7. Chan Zuckerberg Biohub, San Francisco, CA 94158 USA

8. These authors contributed equally: Xiaoyu Shi, Qi Li

*e-mail: ian.seiple@ucsf.edu \& bo.huang@ucsf.edu 


\begin{abstract}
Expansion microscopy (ExM) improves the resolution of fluorescence microscopy by physically expanding the sample embedded in a hydrogel ${ }^{1-4}$. Since its invention, ExM has been successfully applied to a wide range of cell, tissue and animal samples ${ }^{2-9}$. Still, fluorescence signal loss during polymerization and digestion limits molecular-scale imaging using ExM. Here we report the development of label-retention ExM (LR-ExM) with a set of trifunctional anchors that not only prevent signal loss but also enable high-efficiency protein labeling using enzymatic tags. We have demonstrated multicolor LR-ExM for a variety of subcellular structures. Combining LR-ExM with super-resolution Stochastic Optical Reconstruction Microscopy (STORM), we have achieved $5 \mathrm{~nm}$ resolution in the visualization of polyhedral lattice of clathrin-coated pits in situ.
\end{abstract}


By physically expanding the sample before image acquisition, ExM has enabled the use of a conventional confocal microscope to achieve $\sim 70 \mathrm{~nm}$ lateral spatial resolution ${ }^{2-4,6}$, 9, 10. Recent efforts have further enhanced the resolution of ExM either by increasing the volume expansion ratio ${ }^{11,12}$ or by combining ExM with super-resolution microscopy such as Structured Illumination Microscopy (SIM) 5, 8, 13 and Stimulated Emission Depletion (STED) microscopy ${ }^{7,14,15}$. In all these cases, the homogenization of the specimen through either protease digestion ${ }^{10}$ or protein denaturation ${ }^{2,3}$ is essential to achieve isotropic expansion without structural distortion. To retain the spatial information of the target structures, the biomolecules of interest (e.g. protein ${ }^{2-4}$, RNA ${ }^{1}$ ) and/or labels (e.g. dye-labeled DNA ${ }^{10}$, dye-labeled antibodies ${ }^{2,4}$ or fluorescent proteins ${ }^{4}$ ) are anchored to the hydrogel matrix prior to digestion or denaturation. Nevertheless, digestion and denaturation cause incompletely anchored proteins or protein fragments to be washed out, the polymerization reaction to make the hydrogel produces free radicals that readily destroy fluorophores, and both factors can damage fluorescent proteins. Consequently, more than $50 \%$ of the target molecules can lose labeling after expansion ${ }^{4}$, which is a major issue of current ExM methods 16,17. This label loss is exacerbated when aiming for higher spatial resolution. First, ensuring isotropic expansion at nanometer scale requires more thorough digestion or denaturation, hence more wash-out. Second, superresolution microscopy often requires certain dyes that do not survive hydrogel polymerization reaction. For example, Alexa Fluor (AF) 647, one of the best fluorophores for STORM, suffers $>90 \%$ loss of the fluorescent molecules after polymerization and digestion ${ }^{4}$. Such a labeling efficiency deficit is particularly detrimental for resolving molecular-scale structures ${ }^{18}$.

Herein we report LR-ExM, a method that solves the signal loss problem with a set of small molecule trifunctional anchors that are inert to polymerization, digestion and denaturation and that can be fluorescently labeled after expansion. We have developed this method not only for immunofluorescence labeling but also for enzymatic tags (e.g. SNAP-tag) that are particularly advantageous in their high labeling efficiency by organic dyes with cognizing ligands ${ }^{19}$. Specifically, we designed and synthesized trifunctional anchors with three arms (Figure 1a): (1) N-hydroxysuccinimide (NHS) for attachment to antibodies, benzylguanine (BG) for SNAP-tag recognition, or benzylcytosine (BC) for CLIP-tag recognition; (2) methacrylamide (MA) group for anchoring to the polymer matrix; (3) biotin or digoxigenin (DIG) as two orthogonal reporter handles for conjugation 
to an organic dye after expansion. We have chosen these functional groups and the molecular scaffold so that the anchors are resistant to both proteinase $\mathrm{K}$ digestion and acrylamide gel polymerization. The two orthogonal reporter handles enable two-color labeling and imaging. The structures and syntheses of the anchors are described in detail in Supplementary Figures 1 and 2 and Supplementary Methods.

With different trifunctional anchors, LR-ExM is compatible with both immunofluorescent and enzymatic tags (Figure 1b). For immunofluorescence, we stained fixed cells with antibodies conjugated to NHS-MA-biotin or NHS-MA-DIG, proceeded with the standard ExM procedure of gel polymerization and proteinase $\mathrm{K}$ digestion, then stained the gel with fluorescently labeled streptavidin (for biotin) and/or anti-DIG antibody before expanding the gel in water and imaging. For labeling with enzymatic tags, the procedure is nearly identical except that fixed cells were directly treated with BG- or BCtrifunctional anchors.

To demonstrate label retention, we compared ExM of U2OS cells immunostained for microtubules and clathrin heavy chain using secondary antibodies conjugated to Alexa Fluor 488 (AF488) dye (following the proExM protocol ${ }^{2}$ ), biotin (proExM followed by postexpansion staining with AF488-streptavidin ${ }^{3}$ ) or NHS-MA-biotin (our LR-ExM). We conjugated streptavidin with an average dye:protein ratio of 1:1 so that the fluorescence level in the two cases can be directly compared. In the microtubule images (Figure 1c-e), on average the LR-ExM generated a fluorescence signal that is $5.8 \pm 0.8$ (mean \pm standard deviation, $\mathrm{N}=3$ ) times as high as that by AF488 antibody (Figure 1f. For quantification methods, see Supplementary Information and Supplementary Figure 3). The biotin-antibody sample generated $1.9 \pm 0.2$ (mean \pm standard deviation, $\mathrm{N}=3$ ) times of the fluorescence signal compared to proExM. Taking these values together, we concluded that out of the $\sim 83 \%$ label loss by proExM, 15\% can be attributed to polymerization reaction and $\sim 68 \%$ to digestion. A similar trend in signal level was observed in the clathrin images (Figures $1 \mathrm{~g}-\mathrm{i}$ ). The fluorescence signal can be further amplified by conjugating multiple fluorophores to streptavidin or anti-DIG antibody, as shown in Figure $1 \mathrm{~g}$ in which the anti-DIG antibody was labeled at a dye:antibody ratio of $20: 1$.

We calibrated the length expansion ratio of our LR-ExM protocol to be 4.5. Fitting the cross-section profiles of microtubules to a Gaussian peak then gave a full width at half 
maximum (FWHM) of $84 \pm 1.3 \mathrm{~nm}$ (mean \pm standard deviation, $\mathrm{N}=3$ independent experiments) after rescaling by the expansion ratio. Taken the size of immunostained microtubules ${ }^{20}$ into the cross-section profile for fitting ${ }^{10,21}$ obtained an effective resolution of $71 \pm 1.6 \mathrm{~nm}$. At this effective resolution, we could resolve the hollow shape of CCPs (Figures 1g-j). We also demonstrated two-color LR-ExM by coimmnunostaining CCPs and microtubules with antibodies conjugated with NHS-MAbiotin and NHS-MA-DIG, respectively (Figures $1 \mathrm{~m}$ ). Similarly, we demonstrated LR-ExM for SNAP-tag and CLIP-tag with BG-MA-Biotin and BC-MA-DIG trifunctional anchors, respectively (Figure $1 \mathrm{~m}$ for CCPs by overexpressing SNAP-clathrin and Figure $1 \mathrm{n}$ for mitochondria by TOMM20-CLIP), including two-color imaging using both enzymatic tags owing to their orthogonality (Figures $1 n-p)$.

To showcase the application of combined enzymatic labeling and immunostaining, we imaged nuclear lamina with SNAP-tag labeled Lamin A/C (LMNA) and antibody-stained nuclear pore complex (NPC) (Figures 2 a-e). Nuclear lamina is a dense fibrillar network bridging the nuclear envelope and chromatin. It positions the nuclear pore complexes ${ }^{22}$ and participates in chromatin organization ${ }^{23,24}$. Two-color LR-ExM cleanly resolved the holes in the nuclear lamina where NPCs are located. The high labeling efficiency of enzymatic tags was the key to achieving superior image quality compared to previous super-resolution microscopy results using antibody staining ${ }^{25}$. The area of the holes in the Lamin A network varies from 0.1 to $0.5 \mu \mathrm{m}^{2}$, with an average of $0.35 \mu \mathrm{m}^{2}$ (Figure 2e), which is in agreement with previous electron microscopy (EM) ${ }^{22}$ and SIM studies ${ }^{25}$. We observed a strong anti-correlation between Lamin A and NPC signal (Figures 2b-d).

We further characterized the spatial relationship between Lamin A/C network and two different chromatin markers: H3K9me3 for repressed chromatin ${ }^{26}$ (Figures 2f-i) and H3K4me3 for active chromatin ${ }^{27}$ (Figures 2j-m). With the enhanced spatial resolution, two-color LR-ExM images clearly showed that, near the nuclear envelop, H3K9me3 was concentrated near Lamin A/C-rich regions, whereas $\mathrm{H} 3 \mathrm{~K} 4 \mathrm{me} 3$ had an anti-correlation with Lamin A/C signal in a similar manner as NPC (Figure $2 n$ ). This result agrees with the model for the lamin-association of heterochromatin and NPC-association of euchromatin at the nuclear periphery. We were also able to resolve the distinct fine network features of Lamin $A / C$ in a variety of cell lines including mouse embryonic stem cells (Figures 2o-r). All of these results illustrate the application of LR-ExM (potentially in conjugation with fluorescence in situ hybridization to visualize DNA) in studying 
chromatin organization and sub-diffraction-limit-sized chromatin domains such as laminassociated domains.

The retention of labels and hence improved fluorescence signal in LR-ExM enhances its combination with super-resolution microscopy to further improve the spatial resolution. We first demonstrated this application by imaging antibody-stained microtubules in U2OS cells using LR-ExM combined with SIM (LR-ExSIM) (Figures 3a-d). The transverse profile of a microtubule in the final image showed two resolved peaks separated by about $40 \mathrm{~nm}$ (Figure 3c), agreeing with the size of antibody-coated microtubules (Figure 3d) previously measured by STORM 20, 21. By fitting the peaks to Gaussian functions, we calculated the resolution (FWHM) of LR-ExSIM to be $34 \mathrm{~nm}$.

Finally, we demonstrated imaging at molecular resolution by combining LR-ExM with STORM (LR-ExSTORM) ${ }^{28}$. For this purpose, we have examined commonly used photoswitchable dyes including Cy5, Cy5.5, and Alexa Fluor 647, all of which show no loss in either molecular brightness or photoswitching kinetics compared to nonexpansion STORM conditions ${ }^{20}$. The expansion ratio was 3.3 due to added mercaptoethylamine as required for dye photoswitching, leading to an effective localization precision of $\sim 5 \mathrm{~nm}$ (FHWM, calculated from the detected photon number), which is comparable to the size of a typical protein molecule. This result was facilitated by a coverglass coating scheme and sample chamber that we devised to mechanically stabilize the expanded gel during image acquisition (see Supplementary Information and Supplementary Figure 5).

In U2OS cells expressing SNAP-tag labeled clathrin light chain B (CLTB) (Figures 3e-f), LR-ExSTORM was able to resolve the lattice vertices of clathrin-coated pits as clusters of localization points (Figures $3 \mathrm{~g}-\mathrm{I}$ ). In images focused at the top of CCPs where the lattice plane is horizontal (Figures $3 g-i)$, we measured pairs of distances from the centroid of one cluster to the centroid of its nearest neighbor. The histogram of these nearest neighbor distances (1102 pairs from 134 CCPs) showed a main peak at $20 \mathrm{~nm}$ and a small shoulder peak at $34 \mathrm{~nm}$ (Figure $3 \mathrm{~m}$ ). The main peak matched the distance between adjacent vertices of clathrin lattice as previously measured by EM ${ }^{29}, 30$, while the shoulder peak matched the distance between every other vertices (Figure $3 e$ ). This agreement confirmed the ability of LR-ExSTORM to faithfully expand protein complexes at the $10-20 \mathrm{~nm}$ scale, possibly attributed to the high degree of isotropic expansion 
conferred by thorough protease digestion while avoiding the associated label loss. Moreover, the histogram also indicates that our labeling efficiency has resulted in a majority of vertices containing at least one labeled CLTB, noting that not all clathrin light chains had SNAP-tag in our case because of the presence of endogenous CLTB and the other clathrin light chain isoform, CLTA.

In summary, LR-ExM is an effective and versatile method to enhance the signal and labeling efficiency of expansion microscopy. Our trifunctional anchors can be applied to both antibody and enzymatic labeling. They can also be integrated into most existing ExM protocols, greatly increasing their signals. Overcoming the bottleneck of label loss, the currently achieved post-expansion resolutions of $\sim 70 \mathrm{~nm}$ with confocal microscopy, $\sim 30 \mathrm{~nm}$ with SIM, $\sim 10 \mathrm{~nm}$ with STED, and $\sim 5 \mathrm{~nm}$ with STORM are suitable to cover a wide range of biological questions at various scales.

\section{ACKNOWLEDGMENTS}

We are grateful about Dr. Dan Xie's contributions to optimization of the deformable mirror, and 3D printing of slide adapters for the STORM microscope. We thank Drs. Ed Boyden and Fei Chen for their help with the proExM protocol, and Drs. Joshua C Vaughan and Aaron Halpern for the help with their ExM protocol. We appreciate Dr. Luke Lavis for his consultation on protein tags, Dr. Xiangpeng Li from Adam Abate Lab, and Dr. Xiao Huang from Tjal Desai Lab at UCSF for their consultation on biochemistry and microfluidics. We thank Eric Simental for transfecting mESC cells. This work was also highly inspired by personal conversations with Drs. David Brown, Juan Guan and Dan Xie, and discussions with all the other Huang lab members. This project is supported by the National Institutes of Health (NIH) Director's New Innovator Award DP2OD008479 and R01GM124334 to B. H., by the NIH Pathway to Independence Award 1K99GM126136 and the UCSF Mary Anne Koda-Kimble Seed Award for Innovation to X.S., and by National Science Foundation for a Graduate Research Fellowship 1650113 to A. A. T.. B. H. is a Chan Zuckerberg Biohub investigator.

\section{AUTHOR CONTRIBUTIONS}

X. S., Q. L., Z. D., I. S., and B. H. designed the experiments and interpreted the results. X. S. designed LR-ExM protocols. X. S., Z. L., X. W. and T. C. imaged samples. Q. L. 
and A. A. T. synthesized the trifunctional anchors, Z. D. did image quantification and antibodies conjugation. S. F. and A. R.-A designed and made plasmids. X.S. drafted the manuscript. B. H., I. S., A. A. T., Z. D., S. F. and X. S. edited the manuscript.

\section{COMPETING INTERESTS}

The authors declare no competing financial interests. 

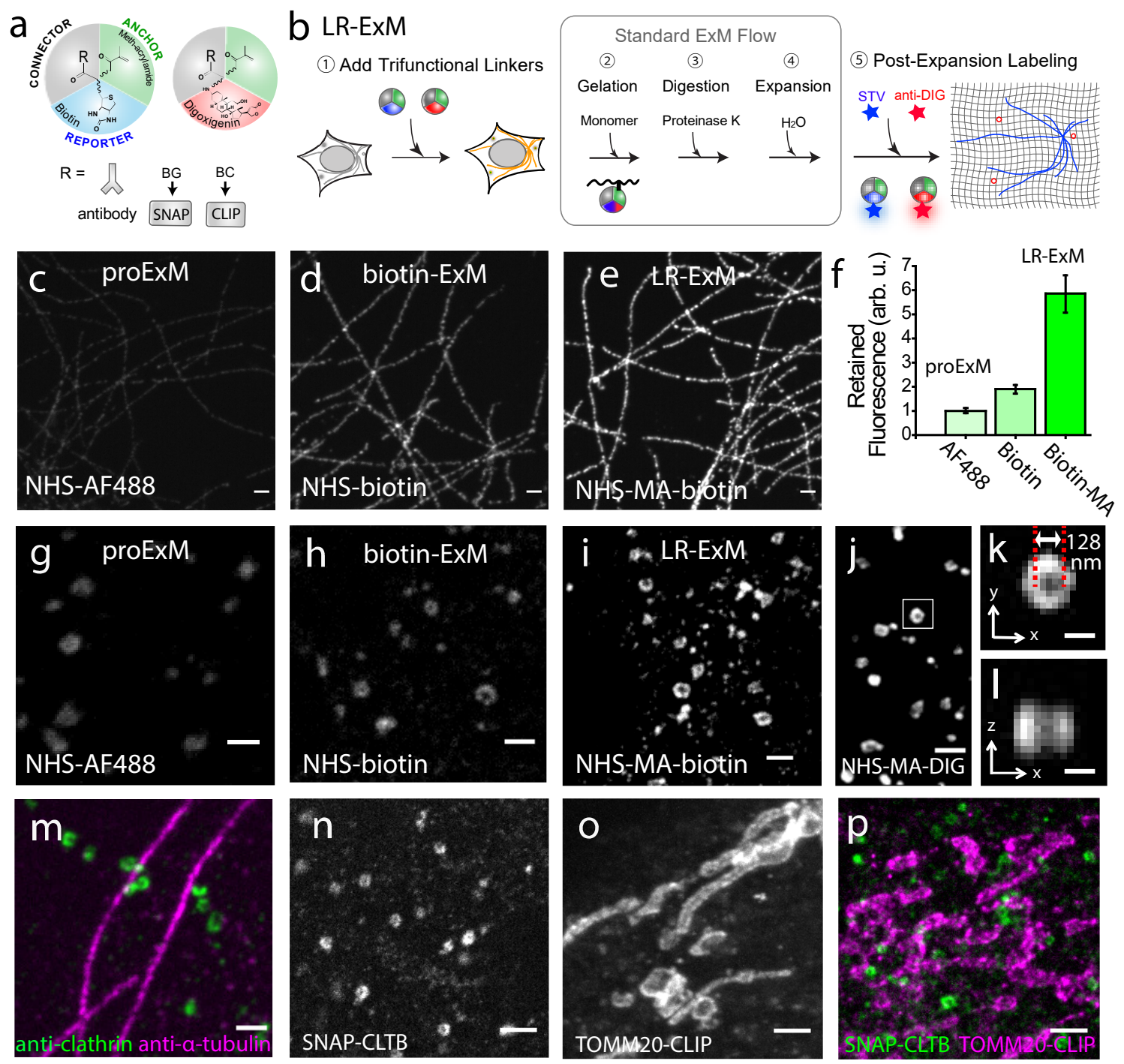

Figure 1 | Immunostaining and protein-tag approaches of LR-ExM. (a) Schematic of trifunctional anchors. (b) Workflow of LR-ExM. (c-e) ExM images of microtubules in U2OS cells. (c) proExM using AF488-conjugated anti- $\alpha$ tubulin antibodies. (d) ExM with post-expansion labeling biotin-conjugated antibodies. (E) LR-ExM using the NHS-MAbiotin tri-functional anchor. (f) Intensity quantification of (c-e). Error bars are standard deviations. $\mathrm{N}=3$ for each case. (g-I) ExM images of CCPs in U2OS cells. (g) proExM using AF488-conjugated anti-clathrin heavy chain antibodies. (h) ExM with postexpansion labeling biotin-conjugated antibodies. (i) LR-ExM using the NHS-MA-biotin trifunctional anchor. (j) LR-ExM using the NHF-MA-DIG anchor. ( $k$ \& I) cross sections of the marked CCP in (j). (m) Two-color LR-ExM of CCP (green) stained with NHS-MADIG, and microtubules (magenta) stained with NHS-MA-biotin. (n-p) LR-ExM images of CCPs and/or mitochondria in HeLa cells stained using protein tags. (n) SNAP-CLTB labeled with BG-MA-biotin. (o) TOMM20-CLIP labeled with BC-MA-biotin. (p) Two-color LR-ExM image of SNAP-CLTB labeled with BG-MA-biotin (green) and TOMM20-CLIP labeled with BC-MA-biotin (magenta). Scale bars, $500 \mathrm{~nm}$ (c-e, g-j, m-p), $100 \mathrm{~nm}$ (k, I). All images taken with a confocal microscope. 

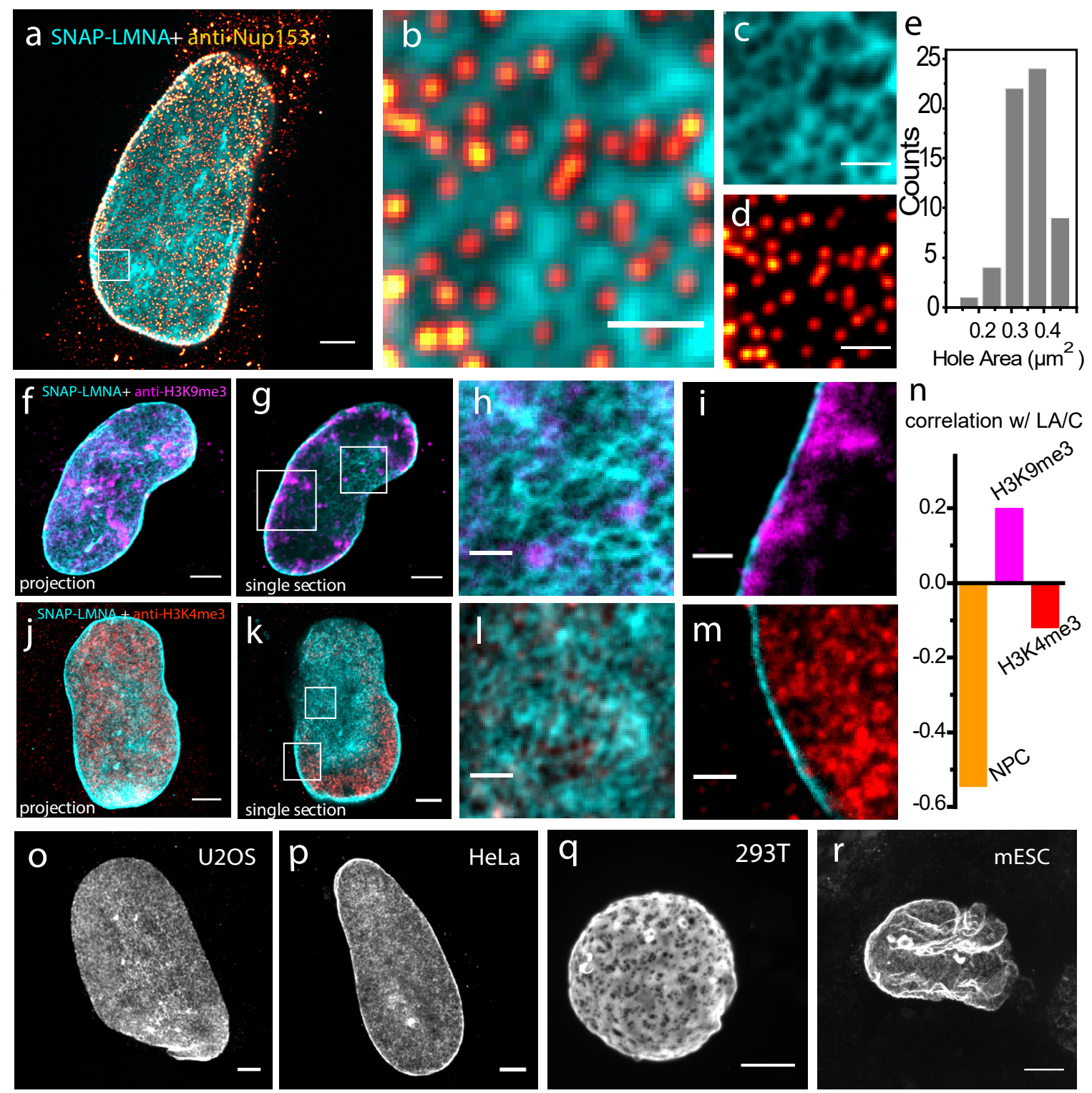

Figure 2 | LR-ExM reveals the spatial relationships of Lamin A with NPC and modified histones. (a) Two-color image of Lamin A/C (cyan) and NPC (red hot). (b) Magnified image of (a) with individual channels shown in (c \& d). (e) histogram of the area of holes in (c). ( $\mathrm{f} \& \mathrm{~g}$ ) Two color images of Lamin A/C (Cyan) and immunostained H3K9me3 (magenta), showing (f) a maximum intensity project of a $z$ stack covering the bottom half of the nucleus and $(\mathrm{g})$ one section of the image stack. ( $\mathrm{h} \& \mathrm{i})$ are magnified images of (g). ( $\mathrm{k}$ k) Two color images of Lamin A/C (Cyan) and immunostained H3K4me3 (red), showing (j) a maximum intensity project of a $z$ stack covering the bottom half of the nucleus and (k) one section of the image stack. (I \& $m$ ) are magnified images of (I). (n) plots the correlation coefficients of NPC with Lamin A/C, H3K9me3 with Lamin A/C, and H3K4me3 with Lamin A/C. (o-r) are the Lamin A/C meshworks of U2OS, HeLa, HEK 293T, and mESC cells, respectively. Each image is a z projection of Lamin A signals over the bottom half of a nucleus. All images were taken with a CSU-W1 spinning-disk confocal microscope. Scale bars: $2 \mu \mathrm{m}$ (a, f, g, j, k, o-r) and $500 \mathrm{~nm}$ (b-d, $h, \mathrm{l}, \mathrm{m}, \mathrm{n})$. 

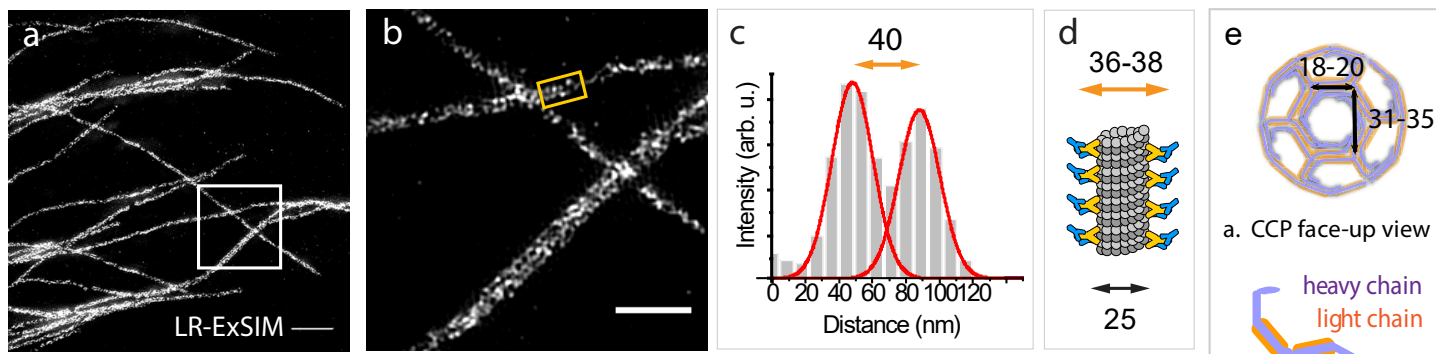

a. CCP face-up view
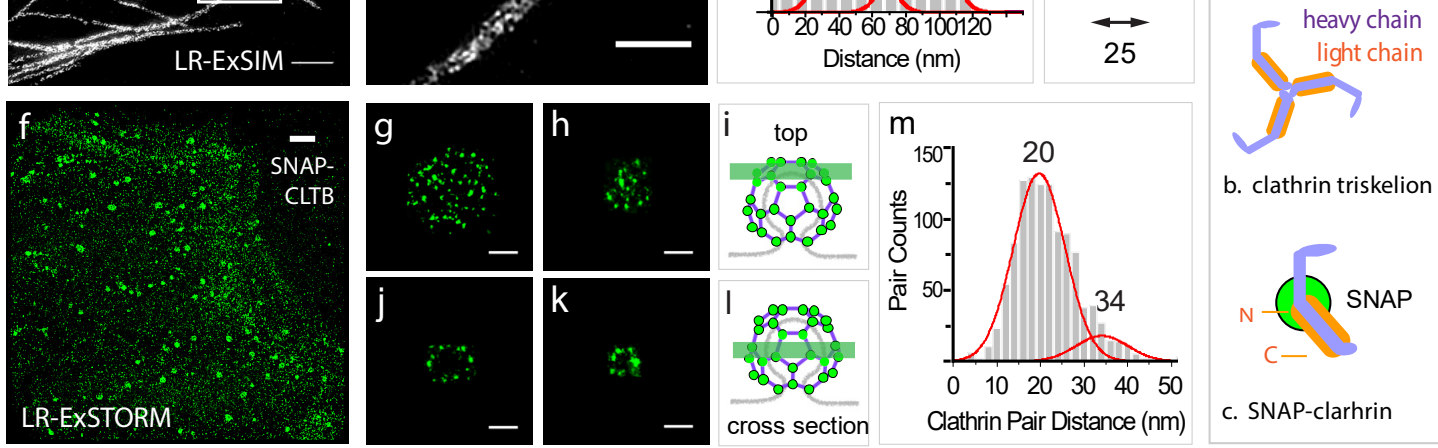

b. clathrin triskelion

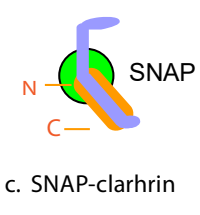

Figure 3 | LR-ExSIM and LR-ExSTORM of cellular structures. (a) LR-ExSIM image of microtubules in a U2OS cell stained with antibody conjugated with NHS-MA-DIG anchors. (b) magnified image of (a). (c) the transverse profile of the microtubule in the gold box in image (b). (d) a schematic of the structure of immunostained microtubule. (e) schematics of the structure of a CCP and with SNAP-tag-labeled CLTB. (f) LRExSTORM image of CCPs in a HeLa cell over expressing SNAP-CLTB, stained with BGMA-biotin, and post-expansion labeled with streptavidin-AF647. (g \& h) images at the top of two CCPs as indicated in (i). ( $\mathrm{j} \& \mathrm{k}$ ) images at the middle of two CCPs as illustrated in (I). (m) Nearest cluster distance analysis of 134 CCPs. Scale bars: 500 nm (a), $1 \mu \mathrm{m}$ (b, f), and $100 \mathrm{~nm}(\mathrm{~g}, \mathrm{~h}, \mathrm{j}, \mathrm{k})$. 


\section{REFERENCES}

1. Chen, F. et al. Nanoscale imaging of RNA with expansion microscopy. Nat Methods 13, 679-684 (2016).

2. Chozinski, T.J. et al. Expansion microscopy with conventional antibodies and fluorescent proteins. Nat Methods 13, 485-488 (2016).

3. $\mathrm{Ku}, \mathrm{T}$. et al. Multiplexed and scalable super-resolution imaging of three-dimensional protein localization in size-adjustable tissues. Nat Biotechnol 34, 973-981 (2016).

4. Tillberg, P.W. et al. Protein-retention expansion microscopy of cells and tissues labeled using standard fluorescent proteins and antibodies. Nat Biotechnol 34, 987992 (2016).

5. Cahoon, C.K. et al. Superresolution expansion microscopy reveals the threedimensional organization of the Drosophila synaptonemal complex. Proc Natl Acad Sci U S A 114, E6857-E6866 (2017).

6. Freifeld, L. et al. Expansion microscopy of zebrafish for neuroscience and developmental biology studies. Proc Natl Acad Sci U S A 114, E10799-E10808 (2017).

7. Gambarotto, D. et al. Imaging cellular ultrastructures using expansion microscopy (U-ExM). Nat Methods 16, 71-74 (2019).

8. Halpern, A.R., Alas, G.C.M., Chozinski, T.J., Paredez, A.R. \& Vaughan, J.C. Hybrid Structured Illumination Expansion Microscopy Reveals Microbial Cytoskeleton Organization. ACS Nano 11, 12677-12686 (2017).

9. Zhang, Y.S. et al. Hybrid Microscopy: Enabling Inexpensive High-Performance Imaging through Combined Physical and Optical Magnifications. Sci Rep 6, 22691 (2016).

10. Chen, F., Tillberg, P.W. \& Boyden, E.S. Optical imaging. Expansion microscopy. Science 347, 543-548 (2015).

11. Chang, J.B. et al. Iterative expansion microscopy. Nat Methods 14, 593-599 (2017).

12. Truckenbrodt, S. et al. X10 expansion microscopy enables $25-\mathrm{nm}$ resolution on conventional microscopes. EMBO Rep 19 (2018). 
13. Wang, $Y$. et al. Combined expansion microscopy with structured illumination microscopy for analyzing protein complexes. Nat Protoc 13, 1869-1895 (2018).

14. Gao, M. et al. Expansion Stimulated Emission Depletion Microscopy (ExSTED). ACS Nano 12, 4178-4185 (2018).

15. Li, R., Chen, X., Lin, Z., Wang, Y. \& Sun, Y. Expansion enhanced nanoscopy. Nanoscale 10, 17552-17556 (2018).

16. Park, Y.G. et al. Protection of tissue physicochemical properties using polyfunctional crosslinkers. Nat Biotechnol (2018).

17. Truckenbrodt, S., Sommer, C., Rizzoli, S.O. \& Danzl, J.G. A practical guide to optimization in X10 expansion microscopy. Nat Protoc 14, 832-863 (2019).

18. Kamiyama, D. \& Huang, B. Development in the STORM. Dev Cell 23, 1103-1110 (2012).

19. Calebiro, D. et al. Single-molecule analysis of fluorescently labeled G-proteincoupled receptors reveals complexes with distinct dynamics and organization. Proc Natl Acad Sci U S A 110, 743-748 (2013).

20. Dempsey, G.T., Vaughan, J.C., Chen, K.H., Bates, M. \& Zhuang, X. Evaluation of fluorophores for optimal performance in localization-based super-resolution imaging. Nat Methods 8, 1027-1036 (2011).

21. Olivier, N., Keller, D., Gonczy, P. \& Manley, S. Resolution doubling in 3D-STORM imaging through improved buffers. PLoS One 8, e69004 (2013).

22. Turgay, Y. et al. The molecular architecture of lamins in somatic cells. Nature 543, 261-264 (2017).

23. Shumaker, D.K., Kuczmarski, E.R. \& Goldman, R.D. The nucleoskeleton: lamins and actin are major players in essential nuclear functions. Curr Opin Cell Biol 15, 358-366 (2003).

24. Zheng, X. et al. Lamins Organize the Global Three-Dimensional Genome from the Nuclear Periphery. Mol Cell 71, 802-815 e807 (2018).

25. Shimi, T. et al. Structural organization of nuclear lamins A, C, B1, and B2 revealed by superresolution microscopy. Mol Biol Cell 26, 4075-4086 (2015). 
26. Nakayama, J., Rice, J.C., Strahl, B.D., Allis, C.D. \& Grewal, S.I. Role of histone H3 lysine 9 methylation in epigenetic control of heterochromatin assembly. Science 292, 110-113 (2001).

27. Liang, G. et al. Distinct localization of histone H3 acetylation and H3-K4 methylation to the transcription start sites in the human genome. Proc Natl Acad Sci U S A 101, 7357-7362 (2004).

28. Tong, Z. et al. Ex-STORM: Expansion Single Molecule Super-resolution Microscopy. bioRxiv 049403, DOI: $10.1101 / 049403$ (2016).

29. Jin, A.J. \& Nossal, R. Rigidity of triskelion arms and clathrin nets. Biophys J 78, 1183-1194 (2000).

30. Kirchhausen, T., Owen, D. \& Harrison, S.C. Molecular structure, function, and dynamics of clathrin-mediated membrane traffic. Cold Spring Harb Perspect Biol 6, a016725 (2014). 


\section{ONLINE METHODS}

Trifunctional anchor synthesis. We synthesized five trifunctional anchors, including HOOC-MA-Biotin, HOOC-MA-DIG, SNAP-MA-Biotin, SNAP-MA-DIG, and CLIP-MA-DIG (Supplementary Figure 1). HOOC-MA-Biotin and HOOC-MA-DIG anchors were converted to NHS-MA-Biotin and NHS-MA-DIG respectively to conjugate antibodies for the immunostaining approach of LR-ExM. The SNAP-MA-Biotin, SNAP-MA-DIG, and CLIP-MA-DIG anchors were directly used for the protein-tag approach of LR-ExM. The synthetic schemes are shown in Supplementary Figure 2.

All reactions were performed in flame- or oven-dried glassware fitted with rubber septa under a positive pressure of nitrogen, unless otherwise noted. All reaction mixtures were stirred throughout the course of each procedure using Teflon-coated magnetic stir bars. Air- and moisture-sensitive liquids were transferred via syringe. Solutions were concentrated by rotary evaporation below $30^{\circ} \mathrm{C}$. Analytical thin-layer chromatography (TLC) was performed using glass plates pre-coated with silica gel $(0.25-\mathrm{mm}, 60-\AA$ pore size, 230-400 mesh, SILICYCLE INC) impregnated with a fluorescent indicator (254 $\mathrm{nm}$ ). TLC plates were visualized by exposure to ultraviolet light (UV) and then were stained by submersion in a basic aqueous solution of potassium permanganate or with an acidic ethanolic solution of anisaldehyde, followed by brief heating. For synthetic procedures and characterization data (thin-layer chromatography (TLC), NMR and Mass Spectroscopy), see Supplementary Methods.

Antibodies. The following primary antibodies were used for the immunostaining approach of LR-ExM: rabbit anti-Clathrin heavy chain antibody (Abcam, ab21679), and rat anti-alpha-Tubulin Antibody, tyrosinated, clone YL1/2 (Millipore Sigma, MAB1864-I). The secondary antibodies used for trifunctional anchor conjugation are: Unconjugated AffiniPure Donkey Anti-Rabbit IgG (H+L) (Jackson ImmunoResearch 711-005-152), and Unconjugated AffiniPure Donkey Anti-Rat IgG (H+L) (Jackson ImmunoResearch 712005-153).

Antibody conjugation. Secondary antibodies were labeled with the amine-reactive trifunctional anchor: NHS-MA-Biotin or NHS-MA-DIG. Amine-reactive trifunctional anchors were freshly made from stock solutions of synthesized trifunctional anchors 
HOOC-MA-Biotin or HOOC-MA-DIG (26 mM, in DMSO, stored at $\left.-20^{\circ} \mathrm{C}\right)$. EDC solution $\left(10 \mu \mathrm{L}, 200 \mathrm{mg} \mathrm{mL}^{-1}\right)$, NHS solution $\left(39 \mu \mathrm{L}, 254 \mathrm{mg} \mathrm{mL}^{-1}\right)$ and DMAP solution $(1 \mu \mathrm{L}, 200$ $\mathrm{mg} \mathrm{mL}^{-1}$ ) were sequentially added into the solution of HOOC-MA-Biotin or HOOC-MADIG (50 $\mu \mathrm{L}, 26 \mathrm{mM})$. The mixture was gently shaken at room temperature for 12 hours while shielding from light with aluminum foil. Using the aforementioned volumes, the final concentration of in situ prepared NHS-MA-Biotin or NHS-MA-DIG is $13 \mathrm{mM}$.

To conjugate the secondary antibodies with the amine-reactive trifunctional anchor, the following procedure was performed: $10 \mu \mathrm{L}$ aqueous $\mathrm{NaHCO}_{3}$ solution $(1 \mathrm{M})$ was added to an Eppendorf tube containing $80 \mu \mathrm{L}$ of unconjugated antibody solution $\left(1 \mathrm{mg} \mathrm{mL}^{-1}\right)$. A solution of the amine-reactive trifunctional anchor (NHS-MA-Biotin or NHS-MA-DIG, 13 $\mathrm{mM}, 24 \mu \mathrm{L}$ ) was then added to the $\mathrm{NaHCO}_{3}$-buffered antibody solution. The labeling reaction mixture was gently rocked for $20 \mathrm{~min}$. During the reaction, a Sephadex G-25 column (GE Healthcare, NAP-5) was equilibrated with PBS ( $\mathrm{pH} 7.4)$. The labeling reaction mixture was loaded onto the column, followed by flowing with $650 \mu \mathrm{L}$ of PBS. The antibodies conjugated with trifunctional anchors were collected by eluting the column with another $250 \mu \mathrm{L}$ of PBS, and stored at $4{ }^{\circ} \mathrm{C}$.

The procedure of antibody conjugation with commercially available bifunctional linker NHS-Biotin was the same to the conjugation with trifunctional anchors, except that a solution of NHS-Biotin ( $26 \mathrm{mM}, 2 \mu \mathrm{L})$ instead of the trifunctional anchor was added to the $\mathrm{NaHCO}_{3}$-buffered antibody solution.

Quantification of biotin-to-antibody ratio. Antibody concentration was characterized by measuring the absorption at $280 \mathrm{~nm}$ with a UV-Vis spectrophotometer. The concentration of biotin was measured using HABA/Avidin reagent kit, following the protocol provided by the supplier (Thermo Scientific ${ }^{\mathrm{TM}}$ Pierce ${ }^{\mathrm{TM}}$ Biotin Quantitation Kit, \# 28005). The biotin-to-antibody ratios of the antibody conjugated with NHS-Biotin (Figure 1d, Supplementary Figure 3b), the antibody conjugated with NHS-MA-Biotin (Figure 1e, Supplementary Figure $3 d$ ), and the antibody conjugated with NHS- Biotin and NHS-MA (Supplementary Figure 3c) are 12.1, 8.6, and 9.9, respectively. The dye-to-antibody ratio of the antibody conjugated with Alexa Fluor 488 (Figure 1c, Supplementary Figure 3a) is 8.9. These biotin-to-antibody ratios and the dye-to-antibody ratio are used to normalize 
the label retention efficiency of proExM, biotin-ExM, and LR-ExM (Figure 1f, Supplementary Figure 3i).

Cell culture. U2OS cells were cultured in McCoy's 5a (ATCC, 30-2007) supplemented with $10 \%$ FBS. HeLa, and HEK 293T cells were cultured in DMEM-Glutamax (Thermo Fisher) supplemented with 10\% FBS. U2OS, HeLa, and HEK 293T cells were seeded at $2.5 \times 10^{4}$ cells $/ \mathrm{cm}^{2}$ in 16-well chambers (Grace Bio-Labs, 112358) and grown to $75 \%$ confluency. Cell lines were not authenticated. No commonly misidentified cell lines were used. All growing cell lines were routinely tested for mycoplasma.

Molecular cloning. To generate the pTOMM20-N-10-CLIPf mammalian expression plasmid, the DNA of CLIPtag was PCR amplified from pCLIPf vector (plasmid source: the Michael Davidson Fluorescent Protein Collection at the UCSF Nikon Imaging Center) using primers (Forward:

GCGGGGATCCACCGGTCGCCACCATGGACAAAGACTGCGAAATGAAGC. Reverse: TCTAGAGTCGCGGCCGCTTAACCCAGCCCAGGCTTGCCC). We then performed restriction enzyme digestion on vector pmEmerald-TOMM20-N-10 (plasmid source: the Michael Davidson Fluorescent Protein Collection at the UCSF Nikon Imaging Center): cutting out the mEmerald sequence between BamHI and Notl. The PCR amplified CLIPtag were then ligated with the digested vectors using In-Fusion HD Cloning kit (Clontech). The plasmids pSNAPf-Clathrin-15 and pSNAPf-LMNA were directly purchased from UCSF Nikon Imaging Center. For constructing the lentiviral production vectors, DNAs of TOMM20-N-10-CLIPf and SNAPf-Clathrin-15 were directly PCR amplified from mammalian expression constructs and subcloned into lentiviral pHRSFFV vector (BamHI/Notl) using In-Fusion HD Cloning kit (Clontech).

Immunostaining. For microtubule immunostaining and microtubule-clathrin coimmunostaining, the cells were fixed with 3.2\% PFA in PEM buffer (100 mM PIPES, 1 mM EGTA, $1 \mathrm{mM} \mathrm{MgCl2,} \mathrm{pH}$ 6.9) at room temperature for $10 \mathrm{~min}$. The fixation was reduced with $0.1 \%$ sodium borohydride in PBS for $7 \mathrm{~min}$. The sodium borohydride was removed by washing with PBS three times with 5 minutes of incubation between washes. The fixed cells were incubated in blocking buffer (PBS, 3\% BSA, 0.1\% Triton X100) for 30 minutes at room temperature. Primary antibodies at a concentration of $2 \mu \mathrm{g}$ $\mathrm{ml}^{-1}$ were added to the fixed cells in blocking buffer for $16 \mathrm{~h}$ at $4{ }^{\circ} \mathrm{C}$. The primary 
antibodies used or this paper are listed in the Supplementary Information. After, primary antibody incubation, the cells were washed with PBS three times with 5 minutes of incubation between washes. Secondary antibodies conjugated with trifunctional anchors were added at a concentration of $3 \mu \mathrm{g} \mathrm{ml}^{-1}$ and incubated for 1 hour in blocking buffer on an orbital shaker. The secondary antibodies were removed by three washes with PBS buffer.

SNAP- and CLIP-tag labeling. The cells expressing SNAP-tag and or CLIP-tag were fixed for 10 minwith 4\% PFA in PBS buffer. The PFA was removed by PBS washing. The fixed cells were incubated in blocking buffer (PBS, 3\% BSA, 0.1\% Triton X-100) for 30 minutes at room temperature. The cells were then incubated in $3 \mu \mathrm{M}$ trifunctional anchor SNAP-MA-Biotin and or $5 \mu \mathrm{M}$ CLIP-MA-DIG for $1 \mathrm{~h}$.

Gelation, proteinase $\mathrm{K}$ digestion, and post-expansion labeling. The gelation and proteinase $\mathrm{K}$ digestion steps are similar to the proExM protocol ${ }^{5}$ with the exception that we also treated the sample with DNAse I prior to gelation to fragment the genomic DNA, with the intention to reduce potential distortions inside and around the nucleus. Specifically, fixed cells were incubated in DNAse I buffer (New Englab BioLabs, M0303S, 1:100 dilution in PBS buffer) for $30 \mathrm{~min}$ at $37^{\circ} \mathrm{C}$, and then were gelated in a mixture of monomer solution (8.6 g Sodium acrylate, $2.5 \mathrm{~g}$ Acrylamide, $0.15 \mathrm{~g} \mathrm{~N}, \mathrm{~N}^{\prime}$ Methylenebisacrylamide, $11.7 \mathrm{~g}$ Sodium chloride per $100 \mathrm{~mL}$ PBS buffer), TEMED (final concentration $0.2 \%(\mathrm{w} / \mathrm{w})$ ) and ammonium persulfate (final concentration $0.2 \%(\mathrm{w} / \mathrm{w})$ ) for $1 \mathrm{~h}$ at $37^{\circ} \mathrm{C}$. The gel was then digested with proteinase $\mathrm{K}$ (Sigma-Aldrich, P4850-5ML) with the final concentration of 8 units $\mathrm{mL}^{-1}$ in digestion buffer $(50 \mathrm{mM}$ Tris $\mathrm{pH} 8.0,1 \mathrm{mM}$ EDTA, $0.5 \%$ Triton $\mathrm{X}-100,0.8 \mathrm{M}$ guanidine $\mathrm{HCl}$ ) for $18 \mathrm{~h}$ at room temperature or $4 \mathrm{~h}$ at $37{ }^{\circ} \mathrm{C}$. After digestion, the proteinase $\mathrm{K}$ was removed by four washes with excessive water, for $30 \mathrm{~min}$ each time. To introduce fluorophores to the trifunctional anchors on the target cellular structures, we incubated the hydrogel in $2 \mu \mathrm{g} \mathrm{mL}^{-1}$ Alexa Fluor 488 labeled Streptavidin (Jackson ImmunoResearch Laboratories, 0165400084) and or DyLight 594 Labeled Anti-Digoxigenin/Digoxin (DIG) (Vector Laboratories, DI-7594) in HEPES buffer (10 mM HEPES, 15m mM NaCl, pH 7.5) for 24 h. For LR-ExSTORM, Alexa Fluor 647 Streptavidin (Jackson ImmunoResearch Laboratories, 0160600084) was used. The post-expansion labeled hydrogel was then washed and expanded by four washes with 
excessive water, at least 30 min each time. It is optional to treat the cells with $25 \mathrm{mM}$ methacrylic acid $\mathrm{N}$-hydroxysuccinimide ester for $1 \mathrm{~h}$ before gelation.

STORM image acquisition and analysis. STORM was performed on a custom-built microscope based on a Nikon Ti-U inverted microscope. Three lasers (Coherent CUBE 405, OBIS 561 and CUBE 642) were combined using dichroic mirrors, aligned, expanded and focused to the back focal plane of the objective (Nikon Plan Apo $\AA \sim 100$ oil NA 1.45). The lasers were controlled directly by the computer. A quad band dichroic mirror (zt405/488/561/640rpc, Chroma) and a band-pass filter (ET705/70m, Chroma) separated the fluorescence emission from the excitation light. During image acquisition, the focusing of the sample was stabilized by a closed-loop system that monitored the back reflection from the sample coverglass via an infrared laser beam sent through the edge of the microscope objective. A low-end piezoelectric deformable mirror (DM) (DMP40-P01, Thorlabs) was added in the emission path at the conjugate plane of the objective pupil plane ${ }^{6}$. By first flattening the mirror and then manually adjusting key Zernike polynomials, this DM corrected aberrations induced by both the optical system and the glass-water refractive index mismatch when the sample was several micrometers away from the coverglass. The fluorescence was recorded at a frame rate of $57 \mathrm{~Hz}$ on an electron multiplying CCD camera (Ixon+ DU897E-CS0-BV, Andor).

The mounting medium used for STORM imaging was water with the addition of $10 \mathrm{mM}$ mercaptoethylamine at $\mathrm{pH} 8.5,5 \%$ glucose $(\mathrm{w} / \mathrm{v})$ and oxygen scavenging enzymes 0.5 $\mathrm{mg} / \mathrm{ml}$ glucose oxidase (Sigma-Aldrich), and $40 \mathrm{mg} / \mathrm{ml}$ catalase (Roche Applied Science). The buffer remained suitable for imaging for 1-2 h. Photoswitchable dye Alexa Fluor 647 was conjugated on streptavidin and was used for imaging with a ratio of 0.8 to 1 dye per streptavidin.

Alexa Fluor 647 was excited with a $642 \mathrm{~nm}$ imaging laser, with typically $1 \mathrm{~kW} \mathrm{~cm}^{-2}$ laser intensity at the focal point. Analysis of STORM raw data was performed in the Insight3 software ${ }^{6}$, which identified and fitted single molecule spots in each camera frame to determine their $\mathbf{x}$ and $\mathbf{y}$ coordinates as well as photon numbers.

Drift reduction and correction. We minimized the sample drift during data acquisition by mounting the hydrogel in a 3D-printed chamber (Supplementary Figure 5). The 
bottom of the chamber is a coverglass modified with poly-L-lysine, which creates a strong adhesion to the negatively charged hydrogel. The drift during data acquisition was further corrected using imaging correlation analysis. The drift-corrected coordinates, photon number, and the frame of appearance of each identified molecule were saved in a molecule list for further analysis.

Quantification and comparison of fluorescence retention efficiencies. To compare the fluorescence retention efficiencies of different ExM methods, we took the images of immunostained microtubules in U2OS cells prepared with different ExM methods, with the same imaging condition. We calculated the retained fluorescence by dividing the total fluorescence intensity of the all microtubules by their total length. The total length of all the microtubules in each image was quantified using a Fiji plugin JFilament. The quantification process and results are shown in Supplementary Figure 3.

Quantification of LR-ExSTORM images of CCPs. We LR-ExSTORM imaged CCPs in U2OS cells expressing SNAP-tag labeled clathrin light chain B (CLTB) stained with BGMA-biotin anchor before expansion and biotin-Alexa Fluor 488 after expansion. CCPs focused at the top were selected for the quantification. We measured the distances from the centroid of one cluster to the centroid of its nearest neighbor in the central area of each CCP, and excluded the clusters at the CCP edge to avoid off-focus localizations. We plotted the histogram of these nearest neighbor distances (1102 pairs from 134 CCPs), and fitted the distance distribution with Gaussian functions. The position of the center and the standard deviation of the gaussian peak are respectively used as the distance between neighboring vertices of the polyhedral CCPs and the standard deviation of the distance (Figure 3).

Data availability. All the images and data are available on request.

Trifunctional anchor availability. Samples of trifunctional anchors described in this manuscript are available on request. 


\section{Additional References}

1. Magano, J. et al. Scalable and Cost-Effective Synthesis of a Linker for Bioconjugation with a peptide and a Monoclonal Antibody. Synthesis 46, 13991406 (2014).

2. Wosnick, J.H., Mello, C. M. \& Swager, T. M. Synthesis and Application of Poly(phenylene Ethynylene)s for Bioconjugation: A Conjugated Polymer-Based Fluorogenic Probe for Proteases. J. Am. Chem. Soc. 127, 3400-3405 (2005).

3. McLaughlin, M., Mohareb, R. M. \& Rapoport, H. An Efficient Procedure for the Preparation of 4-Substituted 5-Aminoimidazoles. J. Org. Chem. 68, 50-54 (2003).

4. Nainar, S. et al. Temporal Labeling of Nascent RNA Using Photoclick Chemistry in Live Cells. J. Am. Chem. Soc. 139, 8090-8093 (2017).

5. Tillberg, P.W. et al. Protein-retention expansion microscopy of cells and tissues labeled using standard fluorescent proteins and antibodies. Nat Biotechnol 34 , 987-992 (2016).

6. Shi, X. et al. Super-Resolution Microscopy Reveals That Disruption of Ciliary Transition-Zone Architecture Causes Joubert Syndrome. Nat Cell Biol 19, no. 1178-1188 (2017). 